\title{
MEMANTINE, AS A P-GLYCOPROTEIN EXPRESSION MODULATOR, ENHANCES LEVETIRACETAM THERAPEUTIC RESPONSE IN EPILEPTIC PATIENTS
}

\author{
SAHAR AHMED HARBY ${ }^{1 *}$, RASHA A NASSRA ${ }^{2}$, JAIDAA F MEKKY ${ }^{3}$, SAMIA M ALI ${ }^{1}$, CHERINE A ISMAIL ${ }^{1}$ \\ ${ }^{1}$ Department of Clinical Pharmacology, Faculty of Medicine, Alexandria University, Alexandria, Egypt. ${ }^{2}$ Department of Medical \\ Biochemistry, Faculty of Medicine, Alexandria University, Alexandria, Egypt. ${ }^{3}$ Department of Neuropsychiatry, Faculty of Medicine, \\ Alexandria University, Alexandria, Egypt. Email: sahar.ahmed15@alexmed.edu.eg
}

Received: 02 February 2020, Revised and Accepted: 12 March 2020

ABSTRACT

Objective: The principal aim of the present study was to assess the importance of multidrug transporter; P-glycoprotein (P-gp) as a potential therapeutic target in patients with epilepsy. Can P-gp transporter expression modulation by memantine add to the standard antiepileptic drugs (AEDs) response?

Methods: A cohort of 56 epilepsy patients was included in a 4 monthly visits prospective study. Patients were on levetiracetam (LEV) 1000 mg/ day alone or combined with other AEDs. They were randomly assigned into two groups; LEV only group including LEV-treated patients and LEV + memantine group including patients on LEV with add-on oral memantine $10 \mathrm{mg} /$ day until the end of the study. During monthly follow-up visits, therapeutic responses were evaluated for each patient by recording the monthly seizures frequency. Blood samples were drawn from every patient twice (on the first and last visits) for assessment of P-gp mRNA expression level.

Results: Fifty patients completed the study. At the end of $4^{\text {th }}$ month, LEV only group showed a non-significant decrease in P-gp expression and seizure frequency compared to the $1^{\text {st }}$ month, whereas, in LEV + memantine group, P-gp expression was significantly reduced and associated with significant seizure control.

Conclusion: Memantine by hindering P-gp overexpression was apt to enhance LEV efficacy and exhibit a better seizure control.

Keywords: P-glycoprotein, N-methyl d-aspartate receptor, Memantine, Levetiracetam, Epilepsy.

(C) 2020 The Authors. Published by Innovare Academic Sciences Pvt Ltd. This is an open access article under the CC BY license (http://creativecommons. org/licenses/by/4. 0/) DOI: http://dx.doi.org/10.22159/ajpcr.2020.v13i5.37011

\section{INTRODUCTION}

Epilepsy is a chronic brain disease characterized by transient dysfunction of the central nervous system induced by abnormal discharge and threatens human health [1]. Approximately two-thirds of epilepsy patients can achieve satisfactory results through antiepileptic drug (AED) treatment, but the remaining third still cannot control their symptoms in spite of using multiple AED treatments and therefore may develop drug-resistant epilepsy (DRE) [2].

The resistance mechanism of DRE remains unclear. Until then, studies should be directed to deeply assimilate the pharmacological hypothesis that helps to elucidate the neurobiological basis for drug resistance, including the transporter hypothesis $[3,4]$. The transporter hypothesis of DRE refers to the excessive expression of certain blood-brain barrier (BBB) efflux transporters that can pump the drugs out of the brain cells, causing a reduction in the local concentration of AEDs at the epileptic lesion [1]. One of the major drug efflux transporters of concern in DRE is P-glycoprotein (P-gp) or ATP-binding cassette subfamily B member 1 protein [5]. Most AEDs have been documented as P-gp substrates with variable degrees, including levetiracetam (LEV) $[6,7]$.

Preventing seizure-associated transporter upregulation, i.e., transporter modulation, might offer a unique alternate approach to overcome transporter-associated pharmacoresistance. Multiplesignal transduction pathways may be involved in brain efflux transporter upregulation; the most evident is glutamate/N-methyl d-aspartate receptor (NMDA-R)/ cyclooxygenase-2 (COX-2)/prostanoid E receptor 1 (EP1) signaling pathway [8]. In this pathway, seizures induce the neuronal and glial release of glutamate, which signals through the NMDA-R, COX-2, and EP1, resulting in increased expression of P-gp in brain capillaries [9]. In this view, memantine as a non-competitive NMDA-R antagonist is suggested to downregulate glutamate/NMDA-R /COX-2 /EP1 signaling pathway in resistant epilepsy [10]. The principal goal of the present study was to assess the importance of P-gp as a potential therapeutic target in patients with epilepsy. In this context, we aimed to evaluate the impact of memantine, as add-on therapy, on the therapeutic response to LEV in epileptic patients.

\section{METHODS}

The study protocol was performed in compliance with the ethical rules of the Helsinki declaration and was approved by the Local Ethics Committee of School of Medicine, Alexandria University. A written informed consent was provided by all participants involving the inclusion of the study data for research purposes.

\section{Patients' inclusion criteria}

The present work displayed a randomized prospective open-label study. Fifty-six patients with epilepsy with the age range of 18-60 years old were recruited from the epilepsy clinic of El-Hadara University Hospital in Alexandria. They had a distinct diagnosis of various seizure types. Only the patients that were on LEV treatment as a single agent or combined with other AEDs were included in the study.

\section{Patients' exclusion criteria}

Patients presenting with secondary epilepsy, significant co-morbidities, neuropsychiatric disorders, impaired hepatic and renal functions, or taking any neuropsychiatric medications other than AEDs were excluded from the study. Furthermore, pregnant and lactating patients and patients with uncontrolled epilepsy with the variation of their AEDs regimen [11,12]. 
Study design

Starting from January 2018, eligible epilepsy patients were randomly enrolled into one of two groups of 28 patients each; LEV only treated group, in which patients that were taking oral LEV (TIRATAM - Al Andalus Medical Company - Egypt) in a dose of 1000 mg/day with or without other AEDs continued taking their same regimen throughout the study. The second group was the LEV + memantine-treated patients that received oral memantine (ALZIXA - Mash Premiere - Egypt) 10 $\mathrm{mg} /$ day at a fixed time every night add-on their already prescribed LEV in a dose of $1000 \mathrm{mg} /$ day until the end of the study $[13,14]$.

\section{Clinical follow-up and sampling}

During monthly follow-up visits, the therapeutic responses were evaluated for each patient by recording the monthly seizures frequency (seizure calendar) [15]. The P-gp mRNA expression level was assessed twice for each patient on the first and last visits. The first visit sample was considered a baseline sample as it was taken before starting memantine. In this context, $3 \mathrm{~mL}$ of venous blood was withdrawn on EDTA for P-gp mRNA extraction from peripheral blood mononuclear cells (PBMCs) [16]. All samples were frozen at $-80^{\circ} \mathrm{C}$ until analysis. The PBMCs were separated from blood samples by centrifugation for $30 \mathrm{~min}$ at $2500 \mathrm{rpm}$ using $1.5 \mathrm{~mL}$ of Ficoll gradient and then cells were washed 3 times with $\times 1$ cold PBS (pH 7.4) [17]

Assessment of P-gp expression in PBMCs by quantitative real-time polymerase chain reaction (PCR)

Total PBMCs RNA was extracted following the manufacturer's protocol (QIAGEN - Germany) and was used for reverse transcription using the High-Capacity cDNA Reverse Transcription Kit (Thermo Fisher Scientific Inc.). Quantitative PCR was performed starting by amplification of the synthesized cDNAs using PCR related kits [17]. The specific primers sequence for P-gp were GTC TGG ACA AGC ACT GAA A (forward) and AAC AAC GGT TCG GAA GTT T (reverse). (GenBank accession number NM 000927.4) [16]. Verification of the PCR amplification product was performed on a $2 \%$ agarose gel stained with ethidium bromide. To confirm PCR product identity, a melting curve analysis was applied and a negative internal control was run with every PCR. Analysis of data was performed, where the level of expression of P-gp mRNA was determined by the comparative CT method for gene expression relative to the housekeeping gene GAPDH [18]
Statistical analysis

Data were analyzed using IBM SPSS software package version 20.0. The Kolmogorov-Smirnov test was used to verify the normality of distribution. Quantitative data were described using range (minimum and maximum), median, and interquartile range. $\mathrm{p}<0.05$ were considered significant. Wilcoxon signed ranks test was used for abnormally distributed quantitative variables, to compare between two periods. Mann-Whitney test was used for abnormally distributed quantitative variables to compare between two studied groups. Friedman test was used for abnormally distributed quantitative variables to compare between more than two periods and post-hoc test (Dunn's) for pairwise comparisons.

\section{RESULTS}

Patients' demographics and clinical features

Only 50 patients completed the study. The individual type of seizures and their LEV regimen together with their characteristic demographics was described in Table 1. Patients' detailed disposition flow in the study was demonstrated in Fig. 1.

\section{P-gp mRNA expression level}

There was a decrease in the $4^{\text {th }}$-month median P-gp expression level compared to the $1^{\text {st }}$ month in the two patients' groups. This decrease was only significant in LEV + memantine group (Table 2). By comparing the percent change in P-gp expression level of the $4^{\text {th }}$ month versus the $1^{\text {st }}$ month between the two patients' groups, LEV + memantine-treated group showed a non-significant percent change compared to LEV only group (Fig. 2).

\section{Seizure frequency/month}

In LEV only group, no significant change was observed in the median number of seizures during the study. While in LEV + memantinetreated group, the median seizure frequency of the $3^{\text {rd }}$ and $4^{\text {th }}$ months showed a significant decrease compared to the $1^{\text {st }}$ month reaching to a seizure-free status in the $4^{\text {th }}$ month (Table 3 ). By comparing the percent change in seizure frequency of the $4^{\text {th }}$ month versus the $1^{\text {st }}$ month in the two patients' groups, LEV + memantine-treated group showed a significant percent reduction compared to LEV only group (Fig. 3)

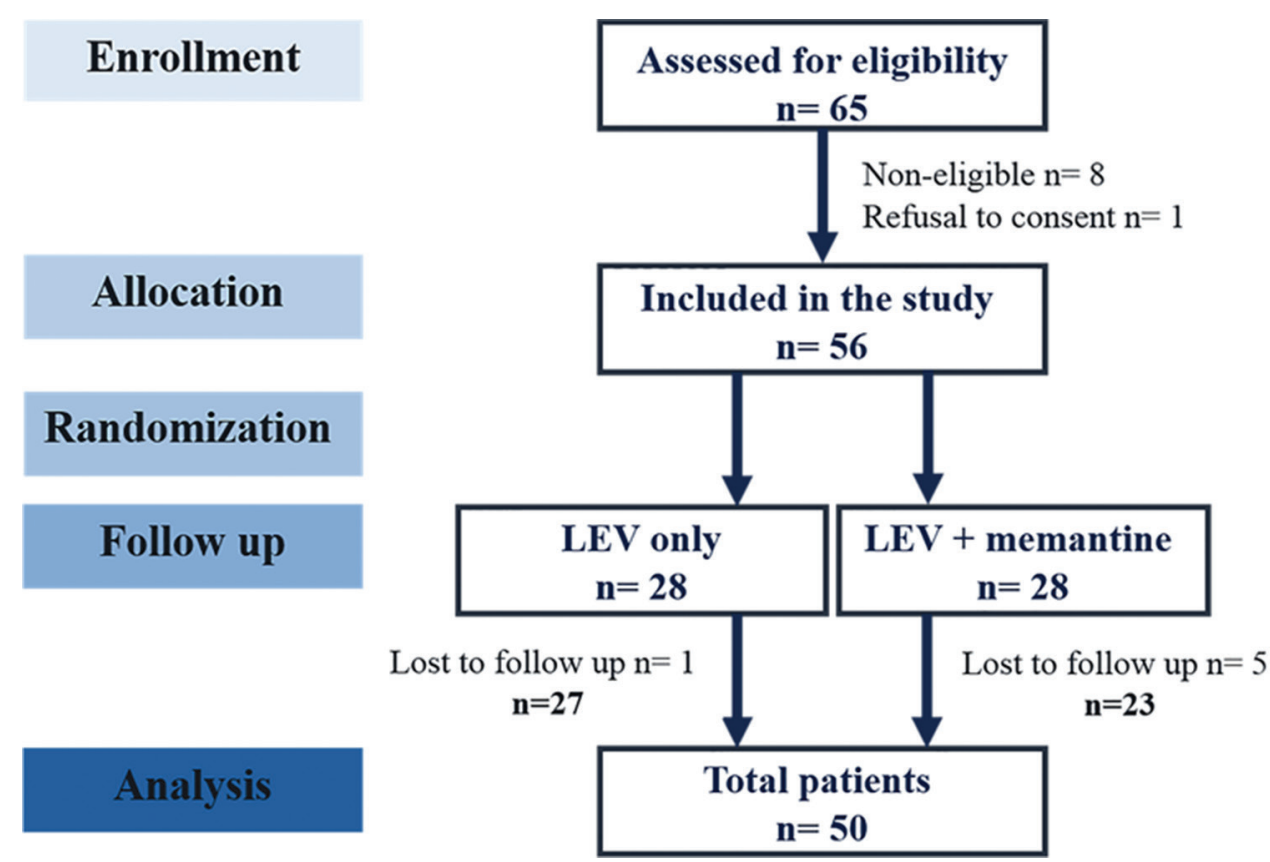

Fig. 1: Patients' disposition flow. Nine patients of 65 were excluded at the beginning of the study because they had one of the exclusion criteria or refused to consent. Only 50 patients of 56 completed the study and were included in the statistical analysis. n: Patients' number; LEV: Levetiracetam 


\section{DISCUSSION}

A concern has been raised highlighting the intimate link of the multidrug transporter P-gp to the transporter hypothesis of pharmacoresistance in epilepsy compared to other BBB efflux transporters [19]. Consequently, the modulation of P-gp could be a plausible therapeutic target in epilepsy. Moreover, unraveling the role of glutamate/NMDA-R/COX-2/ EP1 signaling pathway in the upregulation of the P-gp transporter and its potential link to the transporter hypothesis of AEDs resistance could aid in designing novel and more specific therapeutic strategies targeting this pathway. One of these strategies could be to block NMDA-R by the NMDA-R antagonist memantine.

Results of the present study revealed that the addition of memantine to LEV treatment in epileptic patients had significantly reduced the P-gp expression at the end of the $4^{\text {th }}$ month compared to the baseline, which was lacking in the LEV only treated patients. Furthermore, the effect of memantine on P-gp expression was associated with a significant reduction in the number of seizures at the end of the study compared to the $1^{\text {st }}$ month. The contrast of the seizure percent changes from the $1^{\text {st }}$ to the $4^{\text {th }}$ month between both groups revealed a significant percent reduction in the memantine add-on group versus that of LEV only treated patients.

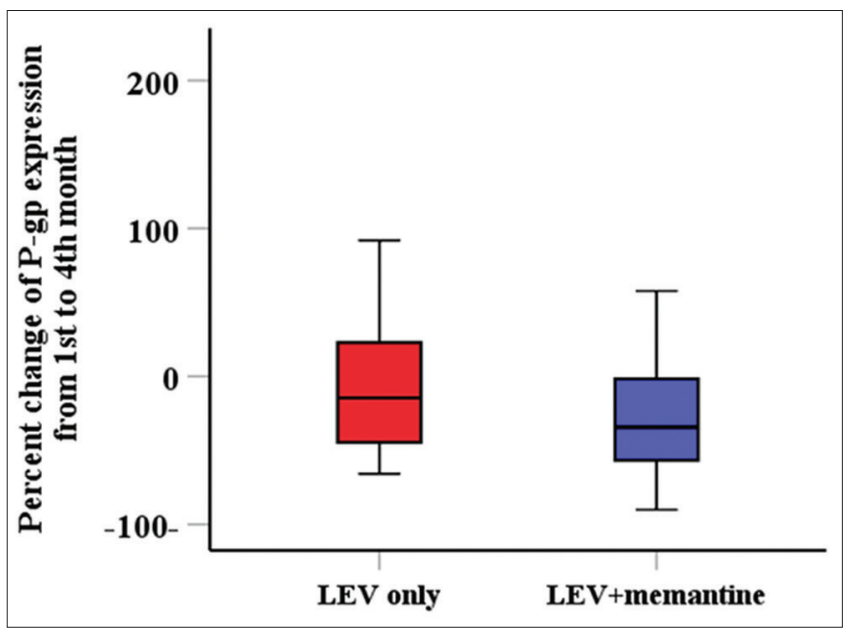

Fig. 2: Percent change of P-glycoprotein expression level from the $1^{\text {st }}$ to the $4^{\text {th }}$ month. LEV: Levetiracetam

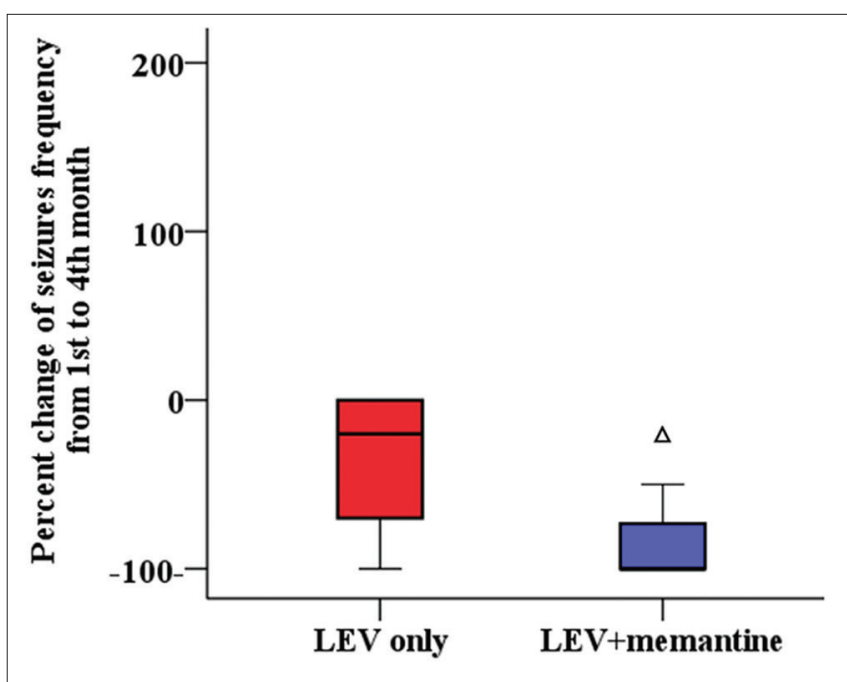

Fig. 3: Percent change of seizure frequency from the $1^{\text {st }}$ to the $4^{\text {th }}$ month LEV: Levetiracetam, ${ }^{\Delta}$ : Significant difference as compared to LEV only group
These results emphasized on the importance of the previously reported role of glutamate/NMDA-R/COX-2/EP1 signaling pathway in pathogenesis of DRE as the blocking of NMDA-R by memantine was apt to halt the downstream signaling pathway and reduced the level of P-gp expression. This effect would increase LEV influx into the target tissues, as evidenced by the improvement in the therapeutic efficacy of LEV to the extent that a significant number of patients turned to be seizure free.

Alternatively, other mechanisms could also be suggested to reason the observed benefit of memantine rather than modulating P-gp expression. One of these mechanisms is the proposed neuroprotective effect of memantine that is speculated to aid in seizure control. Being a non-competitive antagonist at serotonin $5-\mathrm{HT}_{3}$ receptors, memantine can inhibit the promoting effect induced by $5-\mathrm{HT}_{3}^{3}$ receptors stimulation on seizure genesis. It has been reported that $5-\mathrm{HT}_{3}$ receptors facilitate neuronal excitation by transmembrane $\mathrm{Na}^{+}$and $\mathrm{Ca}^{+2}$ influx; thus, their inhibition may inhibit local excitation and spread of seizures. Moreover, $5-\mathrm{HT}_{3}$ receptors blockage has also been reported to diminish the elevated glutamate intracellular levels, oxidative stress, and caspase-3 activity; thus, it may significantly contribute to neuroprotection and seizures reduction $[20,21]$.

Although the use of memantine as add-on AEDs is scarce in literature, the present findings are in accordance with a clinical study on epileptic encephalopathy, where off-label memantine was tried as adjuvant therapy in two children, who were refractory to conventional AEDs. Memantine led to a mild to moderate reduction in seizure burden as well as developmental improvements [22]. It seems likely that the currently observed benefit of memantine can be reproduced by its addition to other AEDs that are P-gp substrates, though this needs further confirmation. Another clinical study was using also memantine in adjuvant to AEDs, yet they were assessing its impact on cognitive

Table 1: Patients' demographics and clinical features

\begin{tabular}{ll}
\hline Demographic data/clinical features & Total $(\mathbf{n}=\mathbf{5 0})$ \\
\hline Sex, $\mathrm{n}(\%)$ & \\
$\quad$ Male & $18(36)$ \\
Female & $32(64)$ \\
Age (years) & \\
$\quad$ Range (median) & $18-60(25.0)$ \\
Types of seizures n (\%) & \\
$\quad$ Generalized & $26(52)$ \\
Focal & $9(18)$ \\
Focal to bilateral tonic clonic & $6(12)$ \\
Myoclonic & $5(10)$ \\
Atonic & $4(8)$ \\
LEV regimen, n (\%) & $14(28)$ \\
LEV monotherapy & $36(72)$ \\
$\quad$ LEV add-on other AEDs & \\
\hline n: Patients' number, LEV: Levetiracetam, AEDs: Antiepileptic drugs, other AEDs \\
include carbamazepine, valproate, phenytoin, and topiramate
\end{tabular}

Table 2: P-glycoprotein expression level of the $4^{\text {th }}$ versus the $1^{\text {st }}$ month

\begin{tabular}{llll}
\hline P-gp expression level & $\mathbf{1}^{\text {st }}$ month & $\mathbf{4}^{\text {th }}$ month & $\mathbf{p}$ \\
\hline LEV only (n=27) & & & \\
$\quad$ Min.-Max. & $0.09-6.29$ & $0.11-5.89$ & 0.079 \\
$\quad$ Median (IQR) & $1.19(0.3-3.5)$ & $1.11(0.4-2.4)$ & \\
LEV+memantine (n=23) & & & \\
$\quad$ Min.-Max. & $0.95-81.80$ & $0.27-80.04$ & $0.004^{* *}$ \\
$\quad$ Median (IQR) & $8.17(4.8-12.7)$ & $4.02(2.5-8.2)$ & \\
\hline
\end{tabular}

$\mathrm{p}$ : Value of significance for Wilcoxon signed ranks test for comparison of

P-gp expression level between the $1^{\text {st }}$ and $4^{\text {th }}$ months. LEV: Levetiracetam, n: Number of patients. Min: Minimum, Max: Maximum, IQR: Interquartile range ${ }^{* *}$ : Statistically significant at $\mathrm{p} \leq 0.01$. P-gp: P-glycoprotein 
Table 3: Monthly seizure frequency

\begin{tabular}{|c|c|c|c|c|c|}
\hline Seizure frequency/month & $1^{\text {st }}$ month & $2^{\text {nd }}$ month & $3^{\text {rd }}$ month & $4^{\text {th }}$ month & $\mathbf{p}$ \\
\hline \multicolumn{6}{|l|}{ LEV only $(n=27)$} \\
\hline Min.-Max. & $0.0-15.0$ & $0.0-30.0$ & $0.0-35.0$ & $0.0-35.0$ & 0.235 \\
\hline Median (IQR) & $3.0(1.0-4.0)$ & $1.0(0.0-3.0)$ & $1.0(0.0-3.0)$ & $2.0(0.0-3.0)$ & \\
\hline \multicolumn{6}{|l|}{ LEV+memantine $(n=23)$} \\
\hline Min.-Max. & 1.030 .0 & $0.0-15.0$ & $0.0-5.0$ & $0.0-2.0$ & $<0.001^{* *}$ \\
\hline Median (IQR) & $1.0(1.0-3.50)$ & $1.0(0.0-3.50)$ & $0.50(0.0-1.0)$ & $0.0(0.0-1.0)$ & \\
\hline $\mathrm{p}_{\text {First }}$ & & 0.114 & $0.001^{* *}$ & $<0.001^{* *}$ & \\
\hline
\end{tabular}

p: Value of significance for Friedman test for comparing seizure frequency between the different 4 monthly visits. $\mathrm{p}_{\text {First }}$ : Value of significance for Dunn's multiple comparisons test for comparison between the $1^{\text {st }}$ month versus each other months. LEV: Levetiracetam, n: Number of patients. Min: Minimum, Max: Maximum,

IQR: Interquartile range ${ }^{* *}$ : Statistically significant at $\mathrm{p} \leq 0.01$

function, memory, and quality of life. They declared that it has a favorable safety profile, which will encourage its safe use in epileptic patients [14].

On experimental level, in line, Schauwecker [23] demonstrated that NMDA-Rs antagonists reduced seizure-induced cell death in a mice model of status epilepticus. It is worth mentioning that the speculated beneficial effect of memantine in seizure control can indirectly downregulate P-gp expression per se. This is based on the evidence declaring that the neuronal injury induced by the seizures can upregulate P-gp expression, which depends on the intensity and the duration of the seizures [24].

It seems that the demonstrated valuable effect of memantine as addon therapy to AEDs in epilepsy is not a group effect of all NMDA-R antagonists. In fact, Sveinbjornsdottir et al. [25] tested the effect of a competitive NMDA-Rs antagonist D-CPP-ene (SDZ EAA 494, (R)-4(3-phosphono-2-propenyl)-2-piperazinecarboxylic acid) on patients with epilepsy. They declared that despite that this compound was well tolerated in healthy volunteers; it induced confusion and sedation in patients with epilepsy with no change or even deterioration of their seizure frequency. This contradiction could not only be due to the difference in structure of the NMDA-R antagonists and in their interaction with the receptors but also due to the difference in clinical setting, where Sveinbjornsdottir et al. [25] tried D-CPP-ene in only eight patients, who were all suffering from refractory epilepsy.

\section{CONCLUSION}

Memantine by hindering the overexpression of P-gp enhanced the antiseizure effect of LEV. The present study provides preliminary clinical proof of the potential impact of P-gp transporter downregulation on seizure control in epilepsy patients. Targeting the glutamate /NMDA-R/ COX-2 /EP1 pathway can pave the way for the future development of safe and effective adjuvant AEDs therapy that can help to overcome the DRE challenge.

\section{AUTHORS' CONTRIBUTIONS}

The author declares that all the named authors have contributed equally to this article.

\section{CONFLICTS OF INTEREST}

The authors have no conflicts of interest to disclose.

\section{FUNDING}

This research did not receive any specific grant from funding agencies.

\section{REFERENCES}

1. Xiong J, Mao DA, Liu LQ. Research progress on the role of ABC transporters in the drug resistance mechanism of intractable epilepsy. Biomed Res Int 2015;2015:194541.

2. Kwan P, Arzimanoglou A, Berg A, Brodie MJ, Allen Hauser W, Mathern G, et al. Definition of drug resistant epilepsy: Consensus proposal by the ad hoc task force of the ILAE commission on therapeutic strategies. Epilepsia 2010;51:1069-77.

3. Tang F, Hartz AM, Bauer B. Drug-resistant epilepsy: Multiple hypotheses, few answers. Front Neurol 2017;8:301.

4. Schiller Y, Najjar Y. Quantifying the response to antiepileptic drugs: Effect of past treatment history. Neurology 2008;70:54-65.

5. Gidal BE. P-glycoprotein expression and pharmacoresistant epilepsy: Cause or consequence? Epilepsy Curr 2014;14:136-8.

6. Potschka H. Modulating P-glycoprotein regulation: Future perspectives for pharmacoresistant epilepsies? Epilepsia 2010;51:1333-47.

7. West CL, Mealey KL. Assessment of antiepileptic drugs as substrates for canine P-glycoprotein. Am J Vet Res 2007;68:1106-10.

8. Bauer B, Hartz AM, Pekcec A, Toellner K, Miller DS, Potschka H. Seizure-induced up-regulation of P-glycoprotein at the blood-brain barrier through glutamate and cyclooxygenase-2 signaling. Mol Pharmacol 2008;73:1444-53.

9. Kambli L, Bhatt LK, Oza M, Prabhavalkar K. Novel therapeutic targets for epilepsy intervention. Seizure 2017;51:27-34.

10. Kumar S. Memantine: Pharmacological properties and clinical uses. Neurol India 2004:52:307-9.

11. Vulliemoz S, Iwanowski P, Landis T, Jallon P. Levetiracetam accumulation in renal failure causing myoclonic encephalopathy with triphasic waves. Seizure 2009;18:376-8.

12. Suresh SH, Chakraborty A, Virupakshaiah A, Kumar N. Efficacy and safety of levetiracetam and carbamazepine as monotherapy in partial seizures. Epilepsy Res Treat 2015;2015:415082.

13. Lancelin F, Franchon E, Kraoul L, Garciau I, Brovedani S, Tabaouti K, et al. Therapeutic drug monitoring of levetiracetam by highperformance liquid chromatography with photodiode array ultraviolet detection: Preliminary observations on correlation between plasma concentration and clinical response in patients with refractory epilepsy. Ther Drug Monit 2007;29:576-83.

14. Marimuthu P, Varadarajan S, Krishnan M, Shanmugam S, Kunjuraman G, Ravinder JR, et al. Evaluating the efficacy of memantine on improving cognitive functions in epileptic patients receiving antiepileptic drugs: A double-blind placebo-controlled clinical trial (Phase IIIb pilot study). Ann Indian Acad Neurol 2016;19:344-50.

15. Zafar A, Shahid R, Nazish S, Aljaafari D, Ali Alkhamis F, Alsalman S, et al. Nonadherence to antiepileptic medications: Still a major issue to be addressed in the management of epilepsy. J Neurosci Rural Pract 2019;10:106-12.

16. Peng XX, Tiwari AK, Wu HC, Chen ZS. Overexpression of P-glycoprotein induces acquired resistance to imatinib in chronic myelogenous leukemia cells. Chin J Cancer 2012;31:110-8.

17. Zhang JC, Xie F, Yu XH, Deng ZY, Wang Y, Liang P, et al. Expression levels of P-glycoprotein in peripheral blood CD8+T lymphocytes from HIV-1-infected patients on antiretroviral therapy. Int J Mol Med 2014;33:431-40.

18. Kosztyu P, Dolezel P, Vaclavikova R, Mlejnek P. Can the assessment of $\mathrm{ABCB} 1$ gene expression predict its function in vitro? Eur J Haematol 2015;95:150-9.

19. Koubeissi M. Neuropathology of the blood-brain barrier in epilepsy: Support to the transport hypothesis of pharmacoresistance. Epilepsy Curr 2013;13:169-71.

20. Rammes G, Danysz W, Parsons C. Pharmacodynamics of Memantine: An update. Curr Neuropharmacol 2008;6:55-78.

21. Mishra A, Goel RK. Chronic 5-HT receptor antagonism ameliorates seizures and associated memory deficit in pentylenetetrazole-kindled mice. Neuroscience 2016;339:319-28.

22. Li D, Yuan H, Ortiz-Gonzalez XR, Marsh ED, Tian L, McCormick EM, et al. GRIN ${ }_{2} \mathrm{D}$ recurrent de novo dominant mutation causes a severe epileptic encephalopathy treatable with NMDA receptor channel 
blockers. Am J Hum Genet 2016;99:802-16.

23. Schauwecker PE. Neuroprotection by glutamate receptor antagonists against seizure-induced excitotoxic cell death in the aging brain. Exp Neurol 2010;224:207-18.

24. Kwan P, Still G, Butler E, Gant T, Meldrum B, Brodie M. Regional expression of multidrug resistance gene in genetically epilepsy-prone rat brain after single ausiogenic sizure. Epilepsia 2002;43:1318-23.

25. Sveinbjornsdottir S, Sander JW, Upton D, Thompson PJ, Patsalos PN, Hirt D, et al. The excitatory amino acid antagonist D-CPP-ene (SDZ EAA-494) in patients with epilepsy. Epilepsy Res 1993;16:165-74. 